\title{
ESTIMATION OF PHYTOPLASMOSIS AND PRODUCTIVITY IN SOME MOLDAVIAN TOMATO VARIETIES
}

\author{
Zamorzaeva Irina, Bahsiev Aighiuni, Mihnea Nadejda \\ Institute of Genetics, Physiology and Plant Protection, Chisinau, Republic of Moldova \\ e-mail: izamorz@gmail.com
}

Phytoplasmosis is a widespread disease of tomato in Moldova. Its distribution in tomato fields is controlled by the climatic conditions of the year, being more or less abundant in different growing seasons. However, this disease is presented in Moldavian tomato varieties every year and has a negative effect on the productivity and quality of fruits. It is known that 'Candidatus Phytoplasma solani' causes the phytoplasmosis in tomatoes in the experimental lots of IGPPP. The degree of damage, as well as the percentage of infected plants, vary from one variety to another. The aim of this study was to detect the spread of ' $\mathrm{Ca}$. P. solani' in the Moldavian tomato varieties Elvira, Cerasus, Desteptarea and Mary Gratefully and to evaluate their productivity and fruits quality during the growing season of 2018. The phytoplasma presence was identified using nested-PCR analysis at DNA isolated from individual plants (10-20 plants in each variety) from the period of mass fruit ripening (August) to the end of the season of vegetation (September). Productivity ( $\mathrm{t} / \mathrm{ha}$ ) and the percentage of marketable fruits were registered. A statistical processing of the data obtained by molecular methods and quantitative measurements were carried out.

The most significant difference in the percentage of infected tomato plants between studied varieties was recorded at the stage of mass fruit ripening. The varieties Elvira and Desteptarea were found to be more susceptible to ' $\mathrm{Ca}$. P. solani' ( $85 \%$ and $80 \%$ of infected plants, respectively) compared with Cerasus (35\% of infected plants) and Mary Gratefully (50\% of infected plants). The difference at the end of the period of vegetation was less pronounced: $80-85 \%$ of plants were infected with phytoplasma in varieties Elvira, Desteptarea and Mary Gratefully; about half of the plants (55\%) were infected in Cerasus.

The variability of the traits of productivity and fruits quality in studied tomato varieties was established in conditions of 2018. In general, varieties that were more resistant to phytoplasma infection, respectively, had a higher yield. Productivity is, firstly, a characteristic of the genotype; secondly, it is influenced by the climatic conditions of the year (changes in temperature, humidity, number of sunny or cloudy days during the growing season, etc.). Diseases of tomato plants other than phytoplasmosis can also affect the productivity and fruit quality. On the other hand, it is known that ' $C a$. P. solani' infection negatively influences on productivity and, especially, reduces the quality of tomato fruits. The correlation between the degree of phytoplasma infection in a variety in August and the yield of marketable fruits was perceptible. Namely, the percentage of marketable fruits in varieties Cerasus and Mary Gratefully that were more resistant to phytoplasma was above $80 \%$. This character in more susceptible to phytoplasma variety Desteptarea consisted of 75\%; most susceptible to phytoplasma infection variety Elvira manifested worst index of the yield of marketable fruits, it was less than $70 \%$.

The correlation between the degree of the phytoplasma presence in tomato field in September, at the end of the growing season, and the yield of marketable fruits in the variety was not significantly pronounced. This fact is not surprising because infection of tomato at the later stages of plants development, after a period of mass fruit ripening, does not affect the quantity and quality of fruits. Tomato harvest mainly takes place in August. Significant spread of ' $\mathrm{Ca}$. P. solani' in a tomato field in September is not important in terms of productivity of varieties and the yield of marketable fruits. This indicator can be useful for the assessing the degree of resistance of tomato varieties to ' $\mathrm{Ca}$. $\mathrm{P}$. solani'. 